\title{
64. SPIRAL WAVES CAUSED BY A PASSAGE OF THE LMC?
}

\section{A. TOOMRE}

Massachusetts Institute of Technology, Cambridge, Mass., U.S.A.

This progress report on the deduction of an almost grazing orbit of the Large Magellanic Cloud from the warped shape of our Galaxy focused on two issues of special relevance to this Symposium:

(i) Distance $R_{0}$. - An implausibly large mass $\left(>3 \times 10^{10} M_{\odot}\right)$ of the LMC is needed to account for the observed warp even with the most optimal orbit, using Schmidt's (1965) disk model of our Galaxy, or any close variant thereof. Although as yet only tentative, the inference seems to be that our distance $R_{0}$ from the galactic center has been overestimated; instead of the now 'standard' $R_{0}=10 \mathrm{kpc}$, these disk-bending calculations suggest a value more like the older $R_{0} \cong 8 \mathrm{kpc}$.

(ii) Forced spiral waves. - Even with the revised $R_{0}$, the sense of the LMC orbit remains ambiguous (though not the perigalactic distance of roughly $20 \mathrm{kpc}$ nor the low absolute inclination of that orbit relative to the galactic plane). Neither the detailed shapes of the vertically bent model disks, nor any computed tidal effects of the Galaxy upon the LMC and SMC, either separately or as a system, rule out an LMC passage that is retrograde with respect to the galactic rotation, but all favor a direct passage as being the more plausible. The latter kind of passage, however, presents an embarrassment of riches. This is best illustrated by Figure 1.

Like the earlier but less specific diagrams of Pfleiderer (1963), it shows the present

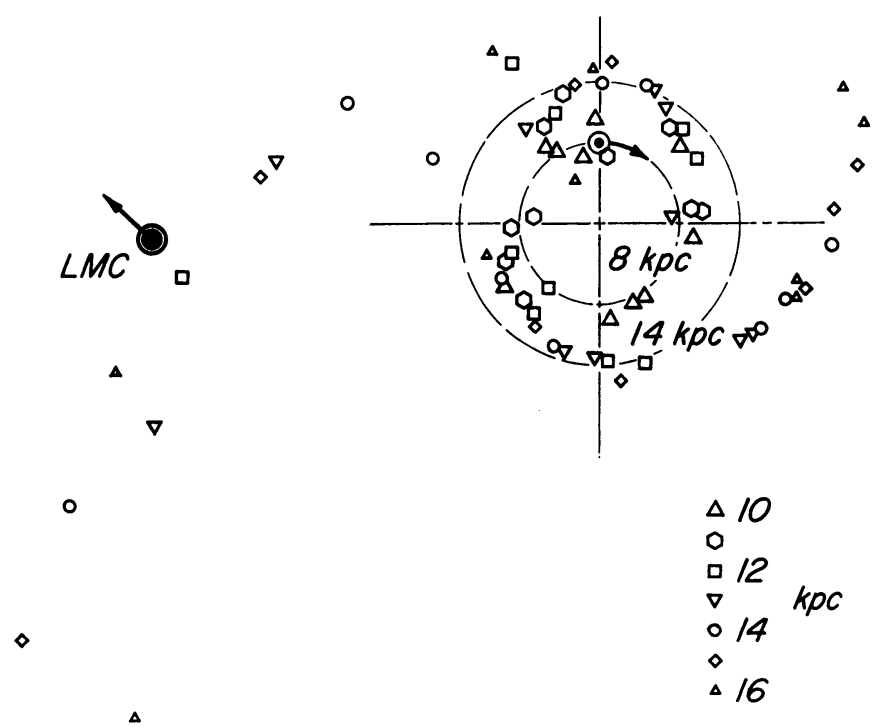

Fig. 1. 
locations (projected onto the galactic plane) of 70 test particles which orbited initially with uniform angular spacing in circles of $10,11, \ldots, 16 \mathrm{kpc}$ radius from a point mass of $8 \times 10^{10} M_{\odot}$ representing the bulk of the Galaxy, and which were then perturbed by the direct, $20 \mathrm{kpc}$ passage of another $1.5 \times 10^{10} M_{\odot}$ particle representing the LMC. Obviously this diagram contains a spectacular 'bridge' of former galactic material towards the LMC, and an opposite feature which resembles (even in latitude) the 'Outer Arm' studied especially by Habing (1966). But it is the vehemence of these phenomena that is alarming: Although it now seems clear from group velocity considerations (Toomre, 1969) that the neglected interactions between the actual disk particles should have caused some of these outer vibrations to propagate inward as a transient spiral density wave, and would thereby have reduced their amplitudes, the amount or rate of that reduction can be estimated only from properly full-scale calculations. Hence we will probably have to wait until some large $n$-body computations such as those of Prendergast and Miller or Hohl have been adapted to this purpose, before we can either dismiss or accept this suggestion that the present spiral structure of our Galaxy may be significantly indebted to external tidal forces from the not too distant past.

\section{References}

Habing, H. J.: 1966, Bull. Astron. Inst. Netherl. 18, 323.

Pfleiderer, J.: 1963, Z. Astrophys. 58, 12.

Schmidt, M.: 1965, Stars and Stellar Systems 5, 513.

Toomre, A.: 1969, Astrophys. J. 158, 899. 